\title{
On shear-rate dependent relaxation spectra in superposition rheometry: a basis for quantitative comparison/interconversion of orthogonal and parallel superposition moduli
}

\author{
D.J. Curtis ${ }^{\mathrm{a}}$, A.R. Davies ${ }^{\mathrm{b}, *}$ \\ ${ }^{a}$ College of Engineering, Swansea University Bay Campus, Fabian Way, Swansea SA1 \\ $8 E N, U K$. \\ ${ }^{b}$ School of Mathematics, Cardiff University, Senghennydd Road, Cardiff CF24 4AG, UK.
}

\begin{abstract}
In 1971, in a seminal paper, Yamamoto derived integral relationships between dynamic moduli and rate-dependent relaxation spectra, $H\left(\tau, \dot{\gamma}^{2}\right)$, in parallel superposition of oscillatory shear on steady shear flow, where both the flows and deformation gradients exist in the same plane. These integral relationships are more complicated than their counterparts for orthogonal superposition (where the oscillatory and unidirectional flow fields occur in orthogonal planes), since they involve not only the spectrum, but also its derivative with respect to unidirectional shear-rate. Herein we derive (i) expressions for determining rate-dependent relaxation spectra directly from parallel superposition rheometry data and (ii) expressions to convert from parallel to orthogonal dynamic moduli in a stable manner. These results facilitate the physical interpretation of parallel superposition dynamic moduli, and direct model-based comparison of parallel and orthogonal superposition moduli in the study of weak nonlinear response.
\end{abstract}

Keywords: superposition rheology, rate-dependent spectra, parallel and orthogonal dynamic moduli, Kramers-Krönig relations, interconversion

\section{Introduction}

Superposition rheometry was established as a technique for probing the nonlinear rheological properties of complex fluids in the 1960's [1,2], and involves superposition of a small amplitude oscillatory perturbation, of amplitude $\gamma_{0}$ and angular frequency $\omega$, upon a unidirectional flow with constant strain-rate $\dot{\gamma}$. The perturbation may be applied either in parallel with, or orthogonal to,

${ }^{*}$ Corresponding author. Electronic mail: DaviesR@cf.ac.uk 
the bulk flow, these being termed parallel and orthogonal superposition rheometry (PSR and OSR), respectively. The kinematics of the two techniques may be conveniently expressed as follows (see Yamamoto [14]):

$$
\begin{aligned}
& x_{1}(t)=x_{1}\left(t^{\prime}\right)+\left[\dot{\gamma}\left(t-t^{\prime}\right)+a\left(e^{i \omega t}-e^{i \omega t^{\prime}}\right)\right] x_{2}\left(t^{\prime}\right), \\
& x_{2}(t)=x_{2}\left(t^{\prime}\right), \\
& x_{3}(t)=x_{3}\left(t^{\prime}\right)+b\left(e^{i \omega t}-e^{i \omega t^{\prime}}\right) x_{2}\left(t^{\prime}\right),
\end{aligned}
$$

where $a=\gamma_{0}$ and $b=0$ for PSR, whilst for OSR $a=0$ and $b=\gamma_{0}$. These kinematics generate stress responses that also consist of unidirectional and oscillatory components. The oscillatory parts of the stress and strain waveforms may hence be used to define a superposition complex modulus $G_{\|}^{*}(\omega, \dot{\gamma})$ or $G_{\perp}^{*}(\omega, \dot{\gamma})$ (in PSR and OSR), respectively). Here, the subscripts $\|$ and $\perp$ serve to distinguish between the superposition moduli, and the linear complex modulus $G^{*}(\omega)[3]$.

A controlled stress implementation of PSR (termed Controlled Stress Parallel Superposition, CSPS) has recently been used to study the microstructure of incipient gel networks formed under controlled stress unidirectional flow conditions $[4,5,6]$. In such experiments $\dot{\gamma} \rightarrow 0$ as the gel point is approached. This marks the transition between viscoelastic liquid and viscoelastic solid-like behaviour that occurs upon establishment of a sample spanning network. Hence, under these conditions $G_{\|}^{*}$ may be interpreted in the same way as $G^{*}$. However, more generally, i.e. where $\dot{\gamma}>0$, interpretation of $G_{\|}^{*}$ is complicated by a coupling of the unidirectional and oscillatory components (as discussed in section 3, below). As $\dot{\gamma}$ increases away from zero, negative values of $G_{\|}^{\prime}$ are often reported in the literature (e.g. $[1,2,7,8,9,18]$ ), thus preventing the conventional interpretation of $G_{\|}^{\prime}$ and $G_{\|}^{\prime \prime}$ in terms of intracycle energy storage and dissipation, as per their quiescent counterparts. It is also commonly stated that the real and imaginary parts of $G_{\|}^{*}$ do not satisfy the Kramers-Krönig relations.

Such complications do not arise in OSR and the components of $G_{\perp}^{*}$ appear to retain the same physical meaning as those of $G^{*}$, for small perturbation amplitudes [10, 11]. Consequently, despite the ease of implementing PSR experiments on commercial rheometers, OSP has (for the past 20 years) been the preferred methodology. However, such experiments require specific hardware, e.g., the TA Instruments Orthogonal Superposition accessory, which employs the rheometer's normal force transducer to generate the oscillatory component [12], and the availability of relatively large quantities of material (approximately $50 \mathrm{ml}$ ). Further, a recent study of flow induced anisotropy in colloidal gels employed the ratio of $G^{\prime}$ measured in orthogonal directions as a measure of anisotropy [11]. In that study, in order to avoid 'the problems associated with parallel superposition experiments', materials for which microstructural recovery was relatively slow were studied, such that SAOS 
experiments probing the anisotropic rheology could be performed following cessation of the unidirectional flow [11]. There is hence clearly a need for further study of superposition moduli, (i) to allow physical interpretation of $G_{\|}^{*}$, and (ii) facilitate the development of a quantitative interpretation of flow induced anisotropy probed by superposition rheometry [13].

Herein we aim to demonstrate that, for certain Lodge type integral constitutive equations studied by Yamamoto [14], and often cited in discussion of superposition rheometry, (i) the real and imaginary parts of $G_{\|}^{*}$ do satisfy the the Kramers-Krönig relations, and (ii) relationships between the superposition moduli can be derived that may be used as a basis for a quantitative comparison of PSR and OSR data. We begin with an elementary discussion of the properties of the linear relaxation spectrum $H(\tau)$ before discussing shear-rate dependent spectra as defined by Yamamoto.

\section{The linear relaxation spectrum}

In an incompressible shear deformation, Boltzmann's general linear integral model for viscoelastic materials relates the stress $\sigma(t)$ to the strain-rate $\dot{\gamma}(t)$ in the form

$$
\sigma(t)=\int_{-\infty}^{t} G\left(t-t^{\prime}\right) \dot{\gamma}\left(t^{\prime}\right) d t^{\prime}
$$

where $G(t)$ denotes the relaxation modulus, which is a positive, monotonically decreasing, and continuously differentiable function of time. The stress may also be expressed in terms of the memory function of the material, $M(t)$, defined by the first derivative $M(t)=-\dot{G}(t)$. Thus, providing the shear, $\gamma$, vanishes as $t^{\prime} \rightarrow-\infty$,

$$
\sigma(t)=\int_{-\infty}^{t} M\left(t-t^{\prime}\right) \gamma\left(t, t^{\prime}\right) d t^{\prime}
$$

In keeping with the principle of fading memory, [15], $M(t)$ is also monotonically decreasing, so that $\dot{G}(t)$ is monotonically increasing. Bernstein's theorem [16] states that successive derivatives of $G(t)$ of all orders are alternately monotonically increasing and decreasing if and only if $G(t)$ is the Laplace transform of a positive measure. Under this constraint $G(t)$ is said to be completely monotone and may be written in the form

$$
G(t)=G_{e}+\int_{0}^{\infty} H(\tau) e^{-\frac{t}{\tau}} \frac{d \tau}{\tau}=G_{e}+\mathcal{L}[\tau H(\tau)](t),
$$

where $\mathcal{L}$ denotes Laplace transformation with respect to the variable $\tau^{-1}$, and $G_{e}$ is a material constant given by

$$
G_{e}=\lim _{t \rightarrow \infty} G(t) .
$$


$H(\tau)$ is the relaxation spectrum associated with a continuous or discrete distribution of relaxation times $\tau$. The memory function $M(t)$ may be written as

$$
M(t)=\int_{0}^{\infty} \frac{H(\tau)}{\tau} e^{-\frac{t}{\tau}} \frac{d \tau}{\tau}=\mathcal{L}[H(\tau)](t)
$$

and either equation (1.3) or (1.4) serves as a mathematical definition of the relaxation spectrum. Taking inverse Laplace transforms, one finds

$$
H(\tau)=\tau^{-1} \mathcal{L}^{-1}[G(t)](\tau)=\mathcal{L}^{-1}[M(t)](\tau)
$$

The spectrum is more often recovered from dynamic measurements of the complex modulus, which is defined as a function of frequency, $\omega$, by

$$
G^{*}(\omega)=G^{\prime}(\omega)+i G^{\prime \prime}(\omega)=G_{e}+i \omega \int_{0}^{\infty}\left[G(t)-G_{e}\right] e^{-i \omega t} d t
$$

where $G^{\prime}$ and $G^{\prime \prime}$ denote the storage and loss moduli, respectively. These moduli are related to the spectrum by the pair of Fredholm integral equations

$$
\begin{aligned}
G^{\prime}(\omega)-G_{e} & =\int_{0}^{\infty} \frac{\omega^{2} \tau^{2}}{1+\omega^{2} \tau^{2}} H(\tau) \frac{d \tau}{\tau} \equiv(\mathcal{T H} H)(\omega), \\
G^{\prime \prime}(\omega) & =\int_{0}^{\infty} \frac{\omega \tau}{1+\omega^{2} \tau^{2}} H(\tau) \frac{d \tau}{\tau} \equiv(\mathcal{S H})(\omega)
\end{aligned}
$$

The integral operators $\mathcal{T}$ and $\mathcal{S}$ defined in equations 2.7 and 2.8 have special properties which we shall specify and exploit in due course.

Determining $H(\tau)$ from experimental data for $G$ or $G^{\prime}, G^{\prime \prime}$ reflects an exponentially ill-posed inverse problem. Explicit use of inverse transforms is rarely made: rather, a model is chosen for $H$ containing a finite number of parameters, and these parameters determined by fitting the corresponding models for the moduli to the available data. This calls for regularization, either by restricting the number of parameters, or by stabilizing the fit to the data by means of a regularization functional.

Equations 2.7 and 2.8 relate the real and imaginary parts of $G^{*}(\omega)$ via the common spectrum $H(\tau)$. In complex form we have

$$
G^{*}(\omega)=G_{e}+\int_{0}^{\infty} \frac{i \omega \tau}{1+i \omega \tau} H(\tau) \frac{d \tau}{\tau}
$$

from which it may be deduced that, under weak conditions on $H(\tau), G^{*}(\omega)$ as a function of complex frequency is analytic away from the imaginary axis. This enables $G^{*}(\omega)$ to be rewritten as a Cauchy integral. By taking limits after indented contour integration around the simple poles on the imaginary axis, it may be shown that the real and imaginary parts of $G^{*}(\omega)$ satisfy the 
Kramers-Krönig relations (see, for example, Tschoegl [19]).

In tensorial form, the constitutive equation 2.1 is generally assumed to be valid whenever the deformation is sufficiently small or slowly varying, i.e. within the realm of linear viscoelasticity. For larger or more rapid deformations there are a large number constitutive equations to choose from in modelling the responses encountered. In nonlinear viscoelasticity no single constitutive model is known to predict the behaviour of all materials under all flow conditions. It is important to bear this in mind when studying shear-rate dependent relaxation spectra, since there exist shear-rate dependent response spectra which are defined not only by the constitutive model but also by the flow conditions. In this paper we examine certain response spectra arising in superposition rheology, and how they are related to shear-rate dependent relaxation spectra.

\section{Superposition rheometry and rate dependent relaxation spectra}

In what follows, the dependence of $G_{\|}^{*}$ and $G_{\perp}^{*}$ on $\dot{\gamma}$ will be taken as understood. We consider a relatively simple integral constitutive model of Lodge-type, which has been studied in the context of superposition rheology in the seminal paper by Yamamoto [14] and more recently by Vermant et al [10] and others. In superposition rheology a small amplitude oscillatory shear, $\gamma_{0} e^{i \omega t}$, is superimposed on a steady shear flow (the primary flow) with constant shear-rate $\dot{\gamma}$, either in parallel to the primary flow (PSR) or orthogonal to it (OSR). Experimentally it is less challenging to obtain complex moduli data from PSR than it is from OSR, but the real and imaginary parts of the complex modulus $G_{\perp}^{*}(\omega)=G_{\perp}^{\prime}(\omega)+i G_{\perp}^{\prime \prime}(\omega)$ in OSR are easier to interpret. From the modelling viewpoint, the OSR storage and loss moduli associated with simple Lodge-type models are known to satisfy the Kramers-Krönig relations.

It has been stated several times in the literature that the PSR storage and loss moduli associated with simple Lodge-type models, $G_{\|}^{\prime}(\omega)$ and $G_{\|}^{\prime \prime}(\omega)$, do not satisfy the Kramers-Krönig relations. These statements are assumptions made in consequence of additional first derivative terms which are present in the expressions for the storage and loss moduli. One of the main results in this paper is to prove that, at least for the Lodge-type model given by (3.1) below, these assumptions are false. In this case it is relatively easy to show that $G_{\|}^{\prime}(\omega)$ and $G_{\|}^{\prime \prime}(\omega)$ do satisfy the Kramers-Krönig relations.

Consider a Lodge-type constitutive model given by

$$
\sigma=-p \mathbf{I}+\int_{-\infty}^{t} M\left(t-t^{\prime}, I I_{2 \mathbf{D}}\left(t^{\prime}\right)\right)\left(\mathbf{C}^{-1}\left(t, t^{\prime}\right)-\mathbf{I}\right) d t^{\prime}
$$

where $\mathbf{C}^{-1}\left(t, t^{\prime}\right)$ is the relative Finger strain tensor, $I I_{2 \mathbf{D}}\left(t^{\prime}\right)$ is the second invariant of the rate of deformation tensor $2 \mathbf{D}$ at time $t^{\prime}$, and $M$ is a rate-dependent 
memory function. Following Vermant et al, we define $I I_{2 \mathbf{D}}=\frac{1}{2} \operatorname{tr}\left[(2 \mathbf{D})^{2}\right]$.

The rate-dependent relaxation spectrum for this model is defined in the same way as in 2.5 , i.e. by

$$
H\left(\tau, I I_{2 \mathbf{D}}\left(t^{\prime}\right)\right)=\mathcal{L}^{-1}\left[M\left(t-t^{\prime}, I I_{2 \mathbf{D}}\left(t^{\prime}\right)\right)\right]
$$

where the inverse Laplace transform is taken with respect to $t$. The ratedependent relaxation modulus is then defined by

$$
G\left(t, I I_{2 \mathbf{D}}\left(t^{\prime}\right)\right)=G_{e}(\dot{\gamma})+\mathcal{L}\left[\tau H\left(\tau, I I_{2 \mathbf{D}}\left(t^{\prime}\right)\right)\right],
$$

where $\mathcal{L}$ again denotes Laplace transformation with respect to the variable $\tau^{-1}$.

In steady shear flow we have

$$
I I_{2 \mathbf{D}}=\dot{\gamma}^{2}
$$

whereas with a small amplitude oscillatory shear superimposed, we have

$$
I I_{2 \mathbf{D}}\left(t^{\prime}\right)=\dot{\gamma}^{2}+2 i a \gamma_{0} \dot{\gamma} \omega e^{i \omega t^{\prime}}+O\left(\gamma_{0}^{2}\right),
$$

where the constant $a$ takes the value $a=1$ in PSR and $a=0$ in OSR. Working to first order in $\gamma_{0}$, we follow Yamamoto in expanding $H\left(\tau, I I_{2 \mathbf{D}}\left(t^{\prime}\right)\right)$ about $I I_{2 \mathbf{D}}\left(t^{\prime}\right)=\dot{\gamma}^{2}$ to obtain

$$
H\left(\tau, I I_{2 \mathbf{D}}\left(t^{\prime}\right)\right)=H\left(\tau, \dot{\gamma}^{2}\right)+2 i a \gamma_{0} \dot{\gamma} \omega e^{i \omega t^{\prime}} \frac{\partial}{\partial \dot{\gamma}^{2}} H\left(\tau, \dot{\gamma}^{2}\right)+O\left(\gamma_{0}^{2}\right),
$$

where we have adopted the notation used by Vermant et al [10]

$$
\frac{\partial}{\partial \dot{\gamma}^{2}} H\left(\tau, \dot{\gamma}^{2}\right)=\left[\frac{\partial}{\partial I I_{2 \mathbf{D}}} H\left(\tau, I I_{2 \mathbf{D}}\left(t^{\prime}\right)\right)\right]_{I I_{2 \mathbf{D}}=\dot{\gamma}^{2}}
$$

\subsection{Orthogonal superposition}

In OSR the relevant stress component may be written as

$$
\sigma_{23}(t)=G_{\perp}^{*}(\omega) \gamma_{0} e^{i \omega t}
$$

where, for small amplitudes $\gamma_{0}$, the real and imaginary parts of the complex modulus are given by

$$
\begin{aligned}
G_{\perp}^{\prime}(\omega)-G_{e}(\dot{\gamma}) & =\int_{0}^{\infty} H\left(\tau, \dot{\gamma}^{2}\right) \frac{\omega^{2} \tau^{2}}{1+\omega^{2} \tau^{2}} \frac{d \tau}{\tau}, \\
G_{\perp}^{\prime \prime}(\omega) & =\int_{0}^{\infty} H\left(\tau, \dot{\gamma}^{2}\right) \frac{\omega \tau}{1+\omega^{2} \tau^{2}} \frac{d \tau}{\tau} .
\end{aligned}
$$

We shall assume, in what follows, that the rate-dependent equilibrium modulus $G_{e}(\dot{\gamma})$ is zero, which is the case for viscoelastic liquids. $H\left(\tau, \dot{\gamma}^{2}\right)$ is the 
response spectrum for OSR and must be distinguished from the true relaxation spectrum $H\left(\tau, I I_{2 \mathbf{D}}\left(t^{\prime}\right)\right)$. However, if a mathematical function $\Phi$ can be chosen to represent the response spectrum over the complete range of shearrates: $H\left(\tau, \dot{\gamma}^{2}\right)=\Phi\left(\tau, \dot{\gamma}^{2}\right)$, then it also represents the true spectrum, i.e. $H\left(\tau, I I_{2 \mathbf{D}}\left(t^{\prime}\right)\right)=\Phi\left(\tau, I I_{2 \mathbf{D}}\left(t^{\prime}\right)\right)$. It is not experimentally possible to obtain data to determine the OSR response function over the complete range of shearrates, and so, in this sense, OSR can only partially determine the rate-dependent relaxation spectrum.

\subsection{Parallel superposition}

In PSR the relevant stress component is

$$
\sigma_{12}(t)=\dot{\gamma} \eta(\dot{\gamma})+G_{\|}^{*}(\omega) \gamma_{0} e^{i \omega t},
$$

where the real and imaginary parts of the complex modulus are now given by

$$
\begin{aligned}
& G_{\|}^{\prime}(\omega)=\int_{0}^{\infty}\left[H\left(\tau, \dot{\gamma}^{2}\right) \frac{\omega^{2} \tau^{2}}{1+\omega^{2} \tau^{2}}+\frac{4 \dot{\gamma}^{2} \omega^{2} \tau^{2}}{\left(1+\omega^{2} \tau^{2}\right)^{2}} \frac{\partial}{\partial \dot{\gamma}^{2}} H\left(\tau, \dot{\gamma}^{2}\right)\right] \frac{d \tau}{\tau}, \\
& G_{\|}^{\prime \prime}(\omega)=\int_{0}^{\infty}\left[H\left(\tau, \dot{\gamma}^{2}\right) \frac{\omega \tau}{1+\omega^{2} \tau^{2}}+\frac{2 \dot{\gamma}^{2} \omega \tau\left(1-\omega^{2} \tau^{2}\right)}{\left(1+\omega^{2} \tau^{2}\right)^{2}} \frac{\partial}{\partial \dot{\gamma}^{2}} H\left(\tau, \dot{\gamma}^{2}\right)\right] \frac{d \tau}{\tau} .
\end{aligned}
$$

At first sight, the emergence of the first derivative terms might suggest that the

Kramers-Krönig relations are not satisfied, but closer inspection shows that this is not the case. Using the identities

$$
\begin{aligned}
\tau \frac{\partial}{\partial \tau}\left(\frac{\omega^{2} \tau^{2}}{1+\omega^{2} \tau^{2}}\right) & =\frac{2 \omega^{2} \tau^{2}}{\left(1+\omega^{2} \tau^{2}\right)^{2}}, \\
\tau \frac{\partial}{\partial \tau}\left(\frac{\omega \tau}{1+\omega^{2} \tau^{2}}\right) & =\frac{\omega \tau\left(1-\omega^{2} \tau^{2}\right)}{\left(1+\omega^{2} \tau^{2}\right)^{2}},
\end{aligned}
$$

followed by integration by parts, (see section 3.3 below), equations 3.11 and 3.12 can be rewritten as

$$
\begin{aligned}
G_{\|}^{\prime}(\omega) & =\int_{0}^{\infty}\left[H\left(\tau, \dot{\gamma}^{2}\right)-2 \dot{\gamma}^{2} \tau \frac{\partial^{2}}{\partial \tau \partial \dot{\gamma}^{2}} H\left(\tau, \dot{\gamma}^{2}\right)\right] \frac{\omega^{2} \tau^{2}}{1+\omega^{2} \tau^{2}} \frac{d \tau}{\tau}, \\
G_{\|}^{\prime \prime}(\omega) & =\int_{0}^{\infty}\left[H\left(\tau, \dot{\gamma}^{2}\right)-2 \dot{\gamma}^{2} \tau \frac{\partial^{2}}{\partial \tau \partial \dot{\gamma}^{2}} H\left(\tau, \dot{\gamma}^{2}\right)\right] \frac{\omega \tau}{1+\omega^{2} \tau^{2}} \frac{d \tau}{\tau} .
\end{aligned}
$$

Hence

$$
\begin{aligned}
G_{\|}^{\prime}(\omega) & =\int_{0}^{\infty} H_{\|}\left(\tau, \dot{\gamma}^{2}\right) \frac{\omega^{2} \tau^{2}}{1+\omega^{2} \tau^{2}} \frac{d \tau}{\tau}, \\
G_{\|}^{\prime \prime}(\omega) & =\int_{0}^{\infty} H_{\|}\left(\tau, \dot{\gamma}^{2}\right) \frac{\omega \tau}{1+\omega^{2} \tau^{2}} \frac{d \tau}{\tau},
\end{aligned}
$$


where

$$
H\left(\tau, \dot{\gamma}^{2}\right)-2 \dot{\gamma}^{2} \tau \frac{\partial^{2}}{\partial \tau \partial \dot{\gamma}^{2}} H\left(\tau, \dot{\gamma}^{2}\right)=H_{\|}\left(\tau, \dot{\gamma}^{2}\right)
$$

is the response spectrum for PSR. Comparing 3.17 and 3.18 with 2.7 and 2.8, respectively, we see that $G_{\|}^{\prime}(\omega)$ and $G_{\|}^{\prime \prime}(\omega)$ satisfy the Kramers-Krönig relations. This is the case even if $H_{\|}\left(\tau, \dot{\gamma}^{2}\right)$ takes on both positive and negative values.

\subsection{Integration by parts.}

Let

$$
U=\frac{\omega^{2} \tau^{2}}{1+\omega^{2} \tau^{2}}, \quad V=\frac{\partial}{\partial \dot{\gamma}^{2}} H\left(\tau, \dot{\gamma}^{2}\right) .
$$

Then, using identity 3.13 , it follows that

$$
\int_{0}^{\infty} \frac{2 \omega^{2} \tau^{2}}{\left(1+\omega^{2} \tau^{2}\right)^{2}} \frac{\partial}{\partial \dot{\gamma}^{2}} H\left(\tau, \dot{\gamma}^{2}\right) \frac{d \tau}{\tau}=\int_{0}^{\infty} \tau \frac{\partial U}{\partial \tau} V \frac{d \tau}{\tau}=\int_{0}^{\infty} \frac{\partial U}{\partial \tau} V d \tau .
$$

Integration by parts then gives

$$
\int_{0}^{\infty} \frac{\partial U}{\partial \tau} V d \tau=[U V]_{0}^{\infty}-\int_{0}^{\infty} U \frac{\partial V}{\partial \tau} d \tau
$$

provided the expression on the right is finite. For a polymeric material with no Newtonian solvent, $H$ and all its derivatives vanish as $\tau \rightarrow 0$ and $\tau \rightarrow \infty$. Therefore $\mathrm{V}$ and its derivatives vanish as $\tau \rightarrow 0$ and $\tau \rightarrow \infty$, while $\mathrm{U}$ remains finite. Again, for a material with a Newtonian solvent, $V$ and its derivatives vanish as $\tau \rightarrow 0$ and $\tau \rightarrow \infty$, while $U$ remains finite. Hence in all cases $[U V]_{0}^{\infty}=$ 0. Thus, 3.22 may be rewritten

$$
\int_{0}^{\infty} \frac{\partial U}{\partial \tau} V d \tau=-\int_{0}^{\infty} U \tau \frac{\partial V}{\partial \tau} \frac{d \tau}{\tau}
$$

which gives

$$
\int_{0}^{\infty} \frac{2 \omega^{2} \tau^{2}}{\left(1+\omega^{2} \tau^{2}\right)^{2}} \frac{\partial}{\partial \dot{\gamma}^{2}} H\left(\tau, \dot{\gamma}^{2}\right) \frac{d \tau}{\tau}=-\int_{0}^{\infty}\left[\tau \frac{\partial^{2}}{\partial \tau \partial \dot{\gamma}^{2}} H\left(\tau, \dot{\gamma}^{2}\right)\right] \frac{\omega^{2} \tau^{2}}{1+\omega^{2} \tau^{2}} \frac{d \tau}{\tau}
$$

Substituting 3.24 into 3.11 leads to 3.15 .

The same argument applies to 3.12 , leading to 3.16 . Here, choose

$$
U=\frac{\omega \tau}{1+\omega^{2} \tau^{2}}
$$

with $V$ the same as before. 


\section{Interconversion of parallel and orthogonal response spectra.}

For clarity, let $H_{\perp}$ and $H_{\|}$denote the response spectra for OSR and PSR, respectively. For the Lodge type-models (3.1), the two spectra are related by the linear hyperbolic partial differential equation (3.19). Suppose that $H_{\|}$can be determined from measurement of $G_{\|}^{\prime}$ and $G_{\|}^{\prime \prime}$ for a range of shear-rates $\dot{\gamma}$. Then we can solve (3.19) to determine $H_{\perp}$ and hence $G_{\perp}^{\prime}, G_{\perp}^{\prime \prime}$. Conversely, if $H_{\perp}$ can be determined from measurement of $G_{\perp}^{\prime}$ and $G_{\perp}^{\prime \prime}$, then (3.19) determines $H_{\|}$and hence $G_{\|}^{\prime}, G_{\|}^{\prime \prime}$.

\subsection{Some exact solutions.}

In this section we give local solutions for $H_{\perp}$ in terms of $H_{\|}$, i.e., in the first instance, we restrict attention to a local range of shear-rates $0<\dot{\gamma_{a}}<\dot{\gamma}<\dot{\gamma_{b}}<$ $\infty$, which we denote by $\Gamma_{a b}=\left(\dot{\gamma}_{a}, \dot{\gamma}_{b}\right)$. We anticipate that $\Gamma_{a b}$ covers only part of the measurable range of shear-rates in PSR, but that local solutions can be found for all parts of the range. Note that any function of $\dot{\gamma}^{2}$ is also a function of $\dot{\gamma}$, and so, without loss of generality we may write

$$
\begin{aligned}
H_{\|}\left(\tau, \dot{\gamma}^{2}\right) & =\lambda H(\tau, 0)+\bar{H}_{\|}(\tau, \dot{\gamma}), \\
H_{\perp}\left(\tau, \dot{\gamma}^{2}\right) & =\lambda H(\tau, 0)+\bar{H}_{\perp}(\tau, \dot{\gamma}),
\end{aligned}
$$

where $H(\tau, 0)$ denotes the linear relaxation spectrum, and $\lambda$ is a constant. The shear viscosity is then given by

$$
\eta(\dot{\gamma})=\int_{0}^{\infty} H_{\perp}\left(\tau, \dot{\gamma}^{2}\right) d \tau=\lambda \eta_{0}+\bar{\eta}(\dot{\gamma}), \quad \text { where } \quad \bar{\eta}(\dot{\gamma})=\int_{0}^{\infty} \bar{H}_{\perp}(\tau, \dot{\gamma}) d \tau
$$

where $\eta_{0}$ denotes the zero shear-rate viscosity. No assumption is made regarding the sign of $\bar{\eta}(\dot{\gamma})$. The constant $\lambda$ is chosen to ensure

$$
\eta(\dot{\gamma})=\bar{\eta}(\dot{\gamma})+\lambda \eta_{0} \geq 0, \quad \dot{\gamma}_{a}<\dot{\gamma}<\dot{\gamma}_{b}
$$

We shall require that $\bar{H}_{\perp}(\tau, \dot{\gamma})$ is piecewise continuously differentiable with respect to both variables $\tau$ and $\dot{\gamma}$. This enables the following:

(i) $\bar{H}_{\|}(\tau, \dot{\gamma})$ can be represented by a rate-dependent discrete spectrum; and

(ii) if $\Gamma_{a b}$ is a shear-thinning range, and $\bar{\eta}>0$, we have

$$
\lambda<1 \quad \text { and } \quad \frac{d}{d \dot{\gamma}} \bar{\eta}(\dot{\gamma})=\int_{0}^{\infty} \frac{\partial \bar{H}}{\partial \dot{\gamma}}(\tau, \dot{\gamma}) d \tau<0, \quad \dot{\gamma}_{a}<\dot{\gamma}<\dot{\gamma}_{b} .
$$

On the other hand, if $\bar{\eta}<0$, at least one of the inequalities in 4.5 is reversed.

Since $\partial / \partial \dot{\gamma}^{2}=(2 \dot{\gamma})^{-1} \partial / \partial \dot{\gamma}$, under the decompositions 4.2 and 4.1, equation 3.19 reduces to

$$
-\bar{H}_{\perp}(\tau, \dot{\gamma})+\tau \dot{\gamma} \frac{\partial^{2}}{\partial \tau \partial \dot{\gamma}} \bar{H}_{\perp}(\tau, \dot{\gamma})=-\bar{H}_{\|}(\tau, \dot{\gamma})
$$


Exact solutions to equation 4.6 may be found as follows. Introduce a stretched variable $\xi=\tau \dot{\gamma}^{\alpha}$, where $\alpha$ is a constant. We then seek solutions of the form

$$
\bar{H}_{\perp}(\tau, \dot{\gamma})=\bar{H}_{\perp}(\xi), \quad \text { with } \quad \bar{H}_{\|}(\tau, \dot{\gamma})=\bar{H}_{\|}(\xi), \quad \xi=\tau \dot{\gamma}^{\alpha} .
$$

In the local range of shear-rates $\Gamma_{a b}$, the shear viscosity has the form

$$
\eta(\dot{\gamma})=\lambda \eta_{0}+\kappa \dot{\gamma}^{-\alpha}, \quad \dot{\gamma}_{a}<\dot{\gamma}<\dot{\gamma}_{b},
$$

where $\kappa=\int_{0}^{\infty} \bar{H}_{\perp}(\xi) d \xi$ is a constant. The constants $\alpha$ and $\kappa$ have the same sign if the viscosity over the range $\Gamma_{a b}$ is shear-thinning, but are of opposite sign if the viscosity over this range in shear-thickening.

In terms of the stretched variable $\xi$, equation 4.6 reduces to an ordinary differential equation of second-order:

$$
-\bar{H}_{\perp}(\xi)+\alpha \xi \bar{H}_{\perp}^{\prime}(\xi)+\alpha \xi^{2} \bar{H}_{\perp}^{\prime \prime}(\xi)=-\bar{H}_{\|}(\xi),
$$

where the ${ }^{\prime}$ denotes differentiation with respect to $\xi$. This has a solution for $\bar{H}_{\perp}$ in terms of $\bar{H}_{\|}$given by

$$
\begin{aligned}
\bar{H}_{\perp}(\xi) & =\frac{1}{2} \beta \int^{\xi}\left[\left(\frac{x}{\xi}\right)^{\beta}-\left(\frac{\xi}{x}\right)^{\beta}\right] \bar{H}_{\|}(x) \frac{d x}{x}, \quad \text { with } \quad \beta=1 / \sqrt{\alpha} \\
& =\beta \int^{\ln \xi} \sinh [\beta(\ln x-\ln \xi)] \bar{H}_{\|}(x) d(\ln x)
\end{aligned}
$$

The boundary conditions on $\bar{H}_{\perp}$, which are necessary conditions for the viscosity to remain finite, are

$$
\bar{H}_{\perp}(0)=0, \quad \text { and } \quad \lim _{\xi \rightarrow \infty} \bar{H}_{\perp}(\xi)=0 .
$$

The same conditions 4.12 will be asked of $\bar{H}_{\|}$. Finally, to ensure regularity in equation 4.6 we impose

$$
\lim _{\xi \rightarrow \infty} \xi \bar{H}_{\perp}^{\prime}(\xi)=\lim _{\xi \rightarrow \infty} \xi^{2} \bar{H}_{\perp}^{\prime \prime}(\xi)=0 .
$$

It is clear from 4.10 that, when $\alpha>0$, then $\beta$ is a positive constant, while if $\alpha<0$, then $\beta$ is pure imaginary, with $\beta=-i|\beta|=-i / \sqrt{|\alpha|}$. In the latter case, equation (3.11) may be rewritten as

$$
\bar{H}_{\perp}(\xi)=|\beta| \int^{\ln \xi} \sin [|\beta|(\ln \xi-\ln x)] \bar{H}_{\|}(x) d(\ln x) .
$$

We end this subsection by proving a very interesting result:

Result 4.1. In a shear-thinning region, $\bar{H}_{\|}(\xi)$ must be negative for some values of the relaxation time $\tau$. 
We shall demonstrate later that Result 4.1 allows $G_{\|}^{\prime}(\omega)$ to become negative for a certain range of frequencies $\omega$. As stated in the introduction, there is experimental evidence for this phenomenon reported in the literature. Yamamoto [14] observes that, theoretically, there are conditions under which $G_{\|}^{\prime \prime}(\omega)$ can also become negative for a certain range of frequencies. Our theory does not preclude this eventuality.

To prove Result 4.1, it is enough to consider the case $\alpha>0$, for otherwise $\kappa$ must be negative, and so $\bar{H}_{\|}$must be negative for some values of the relaxation time. Using Liebnitz' rule for integrals to differentiate 4.11, we find

$$
\bar{H}_{\perp}^{\prime}(\xi)=-\beta^{2} \xi^{-1} \int^{\ln \xi} \cosh [\beta(\ln x-\ln \xi)] \bar{H}_{\|}(x) d(\ln x) .
$$

It is clear that if $\bar{H}_{\|}$is everywhere positive then $\xi \bar{H}_{\perp}^{\prime}(\xi)$ is everywhere negative. Furthermore, either there exists a finite constant $C<0$ such that $\xi \bar{H}_{\perp}^{\prime}(\xi) \rightarrow C$ as $\xi \rightarrow \infty$, or $\xi \bar{H}_{\perp}^{\prime}(\xi) \rightarrow-\infty$ as $\xi \rightarrow \infty$. In either case the first condition in 4.13 cannot hold, and so Result 4.1 follows immediately.

\subsection{Discrete and semi-discrete response spectra.}

We may infer from 3.17 and 3.18 that $\bar{H}_{\|}$is representable as a discrete spectrum. However, this spectrum does not share the properties of a linear relaxation spectrum. Result 4.1 informs us that at least one of the discrete modes must carry a negative coefficient. Complete monotonicity of the associated nonlinear relaxation modulus in PSR is thus lost. On the other hand, complete monotonicity is retained by the corresponding OSR relaxation modulus. In this section we explore the nature of a discrete spectral representation for $\bar{H}_{\|}$, and the corresponding spectral representation for $\bar{H}_{\perp}$.

Let us first examine the spectral representation for $\bar{H}_{\perp}$ resulting from a single constituent mode in $\bar{H}_{\|}$. Consider

$$
\bar{H}_{\|}(\xi)=c_{1} \delta\left(\xi-\xi_{1}\right),
$$

where $\delta($.$) is the Dirac point impulse function, and c_{1}, \xi_{1}$ are constants, with $\xi_{1}>0$. The rate-dependence becomes clear by associating with $\xi_{1}$ the ratedependent relaxation time $\tau_{1}=\xi_{1} \dot{\gamma}^{-\alpha}$. Equation 4.16 may then be written

$$
\bar{H}_{\|}(\xi)=c_{1} \dot{\gamma}^{-\alpha} \delta\left(\tau-\tau_{1}\right),
$$

With $\alpha>0, \tau_{1}$ decreases with increasing shear-rate. From 4.11 we deduce

$$
\begin{aligned}
\bar{H}_{\perp}(\xi) & =-\beta c_{1} \xi_{1}^{-1} \sinh \left[\beta\left(\ln \xi-\ln \xi_{1}\right)\right] \mathscr{H}\left(\xi-\xi_{1}\right), \\
& =-\beta c_{1} \xi_{1}^{-1} \sinh \left[\beta\left(\ln \tau-\ln \tau_{1}\right)\right] \mathscr{H}\left(\tau-\tau_{1}\right),
\end{aligned}
$$

where $\mathscr{H}($.$) denotes the Heaviside unit step function.$ 
The response spectrum given by 4.18 is positive for $\xi>\xi_{1}$ provided the coefficient $c_{1}$ is negative. Moreover, with $c_{1}<0$ and $\tau$ fixed, $\bar{H}_{\perp}(\xi)$ is a decreasing function of shear-rate. This means that $H\left(\tau, \dot{\gamma}^{2}\right)$ is also a decreasing function of shear-rate. $\bar{H}_{\perp}(\xi)$ in 4.18 also satisfies the first of the boundary conditions 4.12. However the second boundary condition is not satisfied, since $\left|\bar{H}_{\perp}(\xi)\right| \rightarrow \infty$ as $\xi \rightarrow \infty$. Clearly, therefore, the representation of $\bar{H}_{\|}$by a single discrete mode is not compliant with a finite shear viscosity. We shall show that this situation can easily be rectified by constructing a triplet of Dirac functions with coefficients whose values alternate in sign.

Definition. Let $\xi_{1}, \xi_{2}, \xi_{3}$ be three positive constants with $0<\xi_{1}<\xi_{2}<\xi_{3}$. We define a compliant Dirac triplet as a triplet of the form

$$
D\left(\xi ; \xi_{1}, \xi_{2}, \xi_{3}\right)=c_{1} \delta\left(\xi-\xi_{1}\right)+c_{2} \delta\left(\xi-\xi_{2}\right)+c_{3} \delta\left(\xi-\xi_{3}\right),
$$

where the coefficients $c_{1}, c_{2}, c_{3}$ are chosen in the ratio

$$
c_{1}: c_{2}: c_{3}=\xi_{1} \sinh \left[\beta \ln \left(\frac{\xi_{2}}{\xi_{3}}\right)\right]: \xi_{2} \sinh \left[\beta \ln \left(\frac{\xi_{3}}{\xi_{1}}\right)\right]: \xi_{3} \sinh \left[\beta \ln \left(\frac{\xi_{1}}{\xi_{2}}\right)\right] .
$$

Note that the values of the entries in the ratios on the right-hand side of 4.21 alternate in sign: -,+,--. Also, $\left|c_{2}\right|>\left|c_{1}\right|$. For modelling purposes, each triplet has at most five free parameters: $\beta, c_{1}, \xi_{1}, \xi_{2}, \xi_{3}$, with $\beta$ fixed for the range of shear-rates $\Gamma_{a b}$.

It is now a fairly straightforward exercise to establish the following:

Result 4.2 The compliant Dirac triplet 4.20 has a corresponding orthogonal response spectrum $E\left(\xi ; \xi_{1}, \xi_{2}, \xi_{3}\right)$, which is a hyperbolic spline with knots $\ln \xi_{1}, \ln \xi_{2}, \ln \xi_{3}$. Specifically, $E$ takes the form

$$
E\left(\xi ; \xi_{1}, \xi_{2}, \xi_{3}\right)= \begin{cases}0, & 0 \leq \xi \leq \xi_{1}, \\ -\beta c_{1} \xi_{1}^{-1} \sinh \left[\beta \ln \left(\frac{\xi}{\xi_{1}}\right)\right], & \xi_{1} \leq \xi \leq \xi_{2}, \\ -\beta\left\{c_{1} \xi_{1}^{-1} \sinh \left[\beta \ln \left(\frac{\xi}{\xi_{1}}\right)\right]+c_{2} \xi_{2}^{-1} \sinh \left[\beta \ln \left(\frac{\xi}{\xi_{2}}\right)\right]\right\}, & \xi_{2} \leq \xi \leq \xi_{3}, \\ 0, & \xi \geq \xi_{3} .\end{cases}
$$

It is easy to check that $E \geq 0$ if $c_{1}<0$ and $E \leq 0$ if $c_{1}>0$. Also, $E$ is continuous, with compact support $\left(\xi_{1}, \xi_{3}\right)$. Moreover, the entries on the righthand side of 4.22 for the first three subintervals $\left(0, \xi_{1}\right),\left(\xi_{1}, \xi_{2}\right)$, and $\left(\xi_{2}, \xi_{3}\right)$ follow immediately from 4.18 . When $\xi \geq \xi_{3}$, the entry is

$$
-\beta\left\{c_{1} \xi_{1}^{-1} \sinh \left[\beta \ln \left(\frac{\xi}{\xi_{1}}\right)\right]+c_{2} \xi_{2}^{-1} \sinh \left[\beta \ln \left(\frac{\xi}{\xi_{2}}\right)\right]+c_{3} \xi_{3}^{-1} \sinh \left[\beta \ln \left(\frac{\xi}{\xi_{3}}\right)\right]\right\} .
$$

But for $m=1,2,3$ we may expand the sinh functions as

$$
\sinh \left[\beta \ln \left(\frac{\xi}{\xi_{m}}\right)\right]=\sinh (\beta \ln \xi) \cosh \left(\beta \ln \xi_{m}\right)-\sinh \left(\beta \ln \xi_{m}\right) \cosh (\beta \ln \xi) .
$$


Thus the entry 4.23 vanishes if

$$
\begin{aligned}
c_{1} \xi_{1}^{-1} \cosh \left(\beta \ln \xi_{1}\right)+c_{2} \xi_{2}^{-1} \cosh \left(\beta \ln \xi_{2}\right)+c_{3} \xi_{3}^{-1} \cosh \left(\beta \ln \xi_{3}\right) & =0 \\
\text { and } \quad c_{1} \xi_{1}^{-1} \sinh \left(\beta \ln \xi_{1}\right)+c_{2} \xi_{2}^{-1} \sinh \left(\beta \ln \xi_{2}\right)+c_{3} \xi_{3}^{-1} \sinh \left(\beta \ln \xi_{3}\right) & =0
\end{aligned}
$$

Both these identities are satisfied if the coefficients $c_{1}, c_{2}, c_{3}$ are in the ratio 4.21.

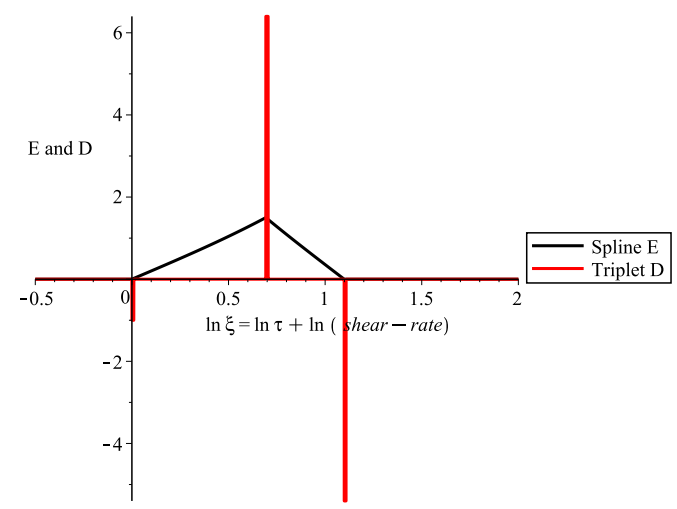

Figure 1: A compliant Dirac triplet, D, and its corresponding hyperbolic spline, E, with $\beta=1 ; c_{1}=-1 ;\left(\xi_{1}, \xi_{2}, \xi_{3}\right)=(1,2,3)$. E is doubled in scale for clarity.

Figure 1 shows a compliant Dirac triplet and its corresponding hyperbolic spline. The parameter values are $\beta=1 ; c_{1}=-1 ;\left(\xi_{1}, \xi_{2}, \xi_{3}\right)=(1,2,3)$. For purposes of illustration, $E$ is doubled in scale for clarity, and the Dirac functions are given finite heights equal to the values of the coefficients. For all shear-rates in the range $\Gamma_{a b}$, the knots $\left(\ln \xi_{1}, \ln \xi_{2}, \ln \xi_{3}\right)$ are fixed. When $\dot{\gamma}=1$, in natural-log scale, the relaxation times $\left(\ln \tau_{1}, \ln \tau_{2}, \ln \tau_{3}\right)$ coincide with the knots, but as $\dot{\gamma}$ increases the relaxation times translate to the left. Viewed as functions of $\ln \tau$, both $D$ and $E$ shift to the left as $\dot{\gamma}$ increases, without changing shape or size. This translational property only applies to the rate-dependent part of $H_{\perp}\left(\tau, \dot{\gamma}^{2}\right)$ and $H_{\|}\left(\tau, \dot{\gamma}^{2}\right)$ in 4.2 and 4.1. If $\lambda \neq 0$, the rate-dependence of $H_{\perp}\left(\tau, \dot{\gamma}^{2}\right)$ and $H_{\|}\left(\tau, \dot{\gamma}^{2}\right)$ is not translational due to the contribution from the linear relaxation spectrum.

Result 4.2 is entirely consistent with the known properties of splines. When a linear differential operator with constant coefficients is applied to a polynomial spline of order $n$, the result is a collection of splines of order $\leq n$. The same is true of hyperbolic splines with a logarithmic variable. Expressed in terms of $\ln \xi$, the differential operator in 4.9 is a linear differential operator with constant coefficients. When applied to the order 2 hyperbolic spline $E$, the result is a collection of hyperbolic splines of order 0 , i.e. the Dirac triplet. 
Recalling the decompositions 4.2 and 4.1, if the linear spectrum $H(\tau, 0)$ is represented as a conventional discrete spectrum and the response spectrum $\bar{H}_{\|}(\tau, \dot{\gamma})$ is represented by Dirac triplets, then the corresponding spectrum $H_{\perp}\left(\tau, \dot{\gamma}^{2}\right)$ will be semi-discrete, i.e. a combination of the discrete linear spectrum and continuous hyperbolic splines of order 2 .

\section{Interconversion of complex moduli.}

There are two ways in which interconversion between $G_{\|}^{*}$ and $G_{\perp}^{*}$ can be accomplished on the basis of the Lodge-type model 3.1. One way is to convert the differential equation 3.19 into a differential equation relating $G_{\perp}^{*}$ to $G_{\|}^{*}$ and finding a solution. In principle, this enables conversion from $G_{\|}^{*}$ to $G_{\perp}^{*}$ directly from the experimental data, without a need for spectral representations $H_{\|}$and $H_{\perp}$. However, the method requires the collection of negative data, which may present challenges in implementation. Also an initial low frequency value for $G_{\perp}^{*}$ is needed, which may be difficult to estimate. Since the method is not without theoretical interest, we will include a brief account below.

The second approach is to use the exact solutions for the response spectra $\bar{H}_{\|}$and $\bar{H}_{\perp}$ obtained in the previous section. It is preferable to have a flow curve available to determine the parameter $\beta$, but this is not strictly necessary. The approach allows interconversion in both directions and necessitates modelfitting. We describe each approach in turn.

\subsection{A differential equation relating $G_{\perp}^{*}$ to $G_{\|}^{*}$.}

The integral operators $\mathcal{T}$ and $\mathcal{S}$ introduced in 2.7 and 2.8 have a special property: they are both anticommutative with respect to homogeneous differentiation. Specifically, let $A(\tau)$ be a continuously differentable function which vanishes at $\tau=0$ and as $\tau \rightarrow \infty$. Then

$$
\left(\mathcal{T} \tau \frac{d}{d \tau} A\right)(\omega)=-\omega \frac{d}{d \omega}(\mathcal{T} A)(\omega) \quad \text { and } \quad\left(\mathcal{S} \tau \frac{d}{d \tau} A\right)(\omega)=-\omega \frac{d}{d \omega}(\mathcal{S} A)(\omega) .
$$

The identities 5.1 are an immediate consequence of integration by parts and the symmetry of the operators $\mathcal{T}$ and $\mathcal{S}$ with respect to the variables $\tau$ and $\omega$. form

For clarity, we restate here the differential equation 3.19 in the equivalent

$$
H_{\perp}\left(\tau, \dot{\gamma}^{2}\right)-\dot{\gamma} \tau \frac{\partial^{2}}{\partial \tau \partial \dot{\gamma}} H_{\perp}\left(\tau, \dot{\gamma}^{2}\right)=H_{\|}\left(\tau, \dot{\gamma}^{2}\right) .
$$

Applying the operator $\mathcal{T}+i \mathcal{S}$ to both sides of 5.2 gives, with the aid of 5.1,

$$
G_{\perp}^{*}(\omega)+\dot{\gamma} \omega \frac{\partial^{2}}{\partial \omega \partial \dot{\gamma}} G_{\perp}^{*}(\omega)=G_{\|}^{*}(\omega) .
$$


The following result follows immediately.

Result 5.1. Since

$$
\lim _{\omega \rightarrow \infty} \omega \frac{\partial^{2} G_{\perp}^{\prime}(\omega)}{\partial \omega \partial \dot{\gamma}}=\lim _{\omega \rightarrow \infty} \int_{0}^{\infty} \frac{2 \omega^{2} \tau^{2}}{\left(1+\omega^{2} \tau^{2}\right)^{2}} \frac{\partial H_{\perp}}{\partial \dot{\gamma}} \frac{d \tau}{\tau}=0,
$$

then the plateau moduli in OSR and PSR, derived from the Lodge-type model 3.1 , are equal, i.e.

$$
\lim _{\omega \rightarrow \infty} G_{\perp}^{\prime}(\omega)=\lim _{\omega \rightarrow \infty} G_{\|}^{\prime}(\omega), \quad \text { or } \quad G_{\perp}^{\prime}(\infty)=G_{\|}^{\prime}(\infty) .
$$

Result 5.1 may also be derived directly from equations 3.11 and 3.8 (with $\left.G_{e}(\dot{\gamma})=0\right)$.

Definition. Two complex-valued functions $G_{\perp}^{*}$ and $G_{\|}^{*}$ satisfying 5.3 are said to be $K K$-compliant if their real and imaginary parts $\left(G_{\perp}^{\prime}, G_{\perp}^{\prime \prime}\right)$ and $\left(G_{\|}^{\prime}, G_{\|}^{\prime \prime}\right)$ both satisfy the Kramers-Krönig relations.

Result 5.2. A sufficient condition for $G_{\perp}^{*}$ and $G_{\|}^{*}$ to be KK-compliant is that there exist response spectra $H_{\perp}$ and $H_{\|}$such that

$$
\begin{aligned}
G_{\perp}^{\prime} & =\mathcal{T} H_{\perp}, \quad G_{\perp}^{\prime \prime}=\mathcal{S} H_{\perp}, \\
\text { and } \quad G_{\|}^{\prime} & =\mathcal{T} H_{\|}, \quad G_{\|}^{\prime \prime}=\mathcal{S} H_{\|} .
\end{aligned}
$$

There is no obvious reason, however, to assume that 5.5 and 5.6 are necessary conditions for KK-compliance. Whereas equation 5.2 implies equation 5.3, the fact that the inverse operators $\mathcal{T}^{-1}$ and $\mathcal{S}^{-1}$ are not, in general, continuous, poses difficulties for the reverse implication. This question is worthy of further investigation, but will not be addressed in this paper.

Clearly, equation 5.3 is of theoretical interest. A solution may be found by proceeding as before. Introduce a reduced frequency variable $\zeta=\omega \dot{\gamma}^{-\alpha}$, where $\alpha>0$ and $\beta=1 / \sqrt{\alpha}$. Now seek a solution of the form

$$
\begin{aligned}
G_{\perp}^{*}(\omega) & =\lambda G^{*}(\omega)+\bar{G}_{\perp}^{*}(\zeta), \\
G_{\|}^{*}(\omega) & =\lambda G^{*}(\omega)+\bar{G}_{\|}^{*}(\zeta),
\end{aligned}
$$

where $G^{*}(\omega)$ is the linear complex modulus $(\dot{\gamma}=0)$, and $\lambda<1$ is a constant chosen in accordance with 4.4. We then obtain

$$
G_{\perp}^{*}(\omega)=\lambda G^{*}(\omega)+\bar{G}_{\perp}^{*}\left(\zeta_{1}\right)+\beta \int_{\ln \zeta_{1}}^{\ln \zeta} \sinh [\beta(\ln z-\ln \zeta)] \bar{G}_{\|}^{*}(z) d(\ln z) .
$$

If an estimate for the low frequency initial value $\bar{G}_{\perp}^{*}\left(\zeta_{1}\right)$ can be found, therefore, equations 5.7 to 5.9 enable conversion from $G_{\|}^{*}$ to $G_{\perp}^{*}$ directly from the experimental data, without a need for spectral representations. However, working 
directly with experimental data in this way does not guarantee KK-compliance of the converted $G_{\perp}^{*}$ and $G_{\perp}^{*}$.

The requirements necessary in the practical implementation of the conversion formula 5.9 are the following:

(i) the collection of possibly negative parallel moduli, $G_{\|}^{*}(\omega)$;

(ii) the estimation of a low frequency initial value $G_{\perp}^{*}\left(\zeta_{1}\right)$;

(iii) sufficiently many sampled frequencies to enable the accurate evaluation of the integrals by numerical quadrature; and

(iv) a flow curve to enable the determination of the parameter $\beta$.

None of these requirements are strictly necessary if spectral representations are chosen for $\bar{G}_{\|}^{*}(\zeta)$ and $G_{\perp}^{*}(\zeta)$. The lower frequency limit $\zeta_{1}$ may then be taken as $\zeta_{1}=0$, with corresponding initial value $\bar{G}_{\perp}^{*}\left(\zeta_{1}\right)=0$, and, at least for rational values of $\beta$, the integrals can be evaluated in closed form, if required.

\subsection{A spectral representation approach.}

As indicated above, working with spectral representations guarantees KKcompliance of the converted $G_{\perp}^{*}$ and $G_{\perp}^{*}$. Consider the spectral decompositions 4.1 and 4.2. Using discrete and semi-discrete representations we have

$$
\begin{aligned}
& H_{\|}\left(\tau, \dot{\gamma}^{2}\right)=\lambda \sum_{k} c_{k 0} \delta\left(\tau-\tau_{k 0}\right)+\sum_{k} D\left(\tau \dot{\gamma}^{\alpha} ; \xi_{k 1}, \xi_{k 2}, \xi_{k 3}\right), \\
& H_{\perp}\left(\tau, \dot{\gamma}^{2}\right)=\lambda \sum_{k} c_{k 0} \delta\left(\tau-\tau_{k 0}\right)+\sum_{k} E\left(\tau \dot{\gamma}^{\alpha} ; \xi_{k 1}, \xi_{k 2}, \xi_{k 3}\right),
\end{aligned}
$$

where the linear spectrum $\left\{c_{k 0}, \tau_{k 0}\right\}$ has been predetermined from a pure oscillatory shear experiment. The corresponding moduli are given by

$$
\begin{aligned}
G_{\|}^{\prime}(\omega) & =\lambda G^{\prime}(\omega)+\bar{G}_{\|}^{\prime}\left(\omega \dot{\gamma}^{-\alpha}\right), \\
& =\lambda \sum_{k} c_{k 0} \frac{\omega^{2} \tau_{k 0}}{1+\omega^{2} \tau_{k 0}^{2}}+\sum_{k} \sum_{m=1}^{3} c_{k m} \frac{\omega^{2} \xi_{k m}}{\dot{\gamma}^{2 \alpha}+\omega^{2} \xi_{k m}^{2}}, \\
G_{\|}^{\prime \prime}(\omega) & =\lambda G^{\prime \prime}(\omega)+\bar{G}_{\|}^{\prime \prime}\left(\omega \dot{\gamma}^{-\alpha}\right), \\
& =\lambda \sum_{k} c_{k 0} \frac{\omega}{1+\omega^{2} \tau_{k 0}^{2}}+\dot{\gamma}^{\alpha} \sum_{k} \sum_{m=1}^{3} c_{k m} \frac{\omega}{\dot{\gamma}^{2 \alpha}+\omega^{2} \xi_{k m}^{2}},
\end{aligned}
$$

and by

$$
\begin{aligned}
G_{\perp}^{\prime}(\omega) & =\lambda G^{\prime}(\omega)+\bar{G}_{\perp}^{\prime}\left(\omega \dot{\gamma}^{-\alpha}\right), \\
& =\lambda \sum_{k} c_{k 0} \frac{\omega^{2} \tau_{k 0}}{1+\omega^{2} \tau_{k 0}^{2}}+\sum_{k}\left[\mathcal{T} E\left(\tau \dot{\gamma}^{\alpha} ; \xi_{k 1}, \xi_{k 2}, \xi_{k 3}\right)\right](\omega), \\
G_{\perp}^{\prime \prime}(\omega) & =\lambda G^{\prime \prime}(\omega)+\bar{G}_{\perp}^{\prime \prime}\left(\omega \dot{\gamma}^{-\alpha}\right), \\
& =\lambda \sum_{k} c_{k 0} \frac{\omega}{1+\omega^{2} \tau_{k 0}^{2}}+\sum_{k}\left[\mathcal{S} E\left(\tau \dot{\gamma}^{\alpha} ; \xi_{k 1}, \xi_{k 2}, \xi_{k 3}\right)\right](\omega) .
\end{aligned}
$$


With $\left\{c_{k 0}, \tau_{k 0}\right\}$ predetermined, the remaining constants $\left\{c_{k m}, \xi_{k m}\right\}$ are determined by fitting the models 5.12 and 5.13 to the available PSR experimental data at a fixed shear-rate $\dot{\gamma}$ in the range $\Gamma_{a b}$. The constants $c_{k 1}, c_{k 2}, c_{k 3}$ must be chosen in the ratio 4.21, so only one in three of these constants is a free parameter.

Note that the models 5.12 to 5.15 are confined to the range of shear-rates $\Gamma_{a b}$, and are not necessarily applicable in the limit $\dot{\gamma} \rightarrow 0$.

To complete the conversion from $G_{\|}^{*}$ to $G_{\perp}^{*}$, it remains only to derive suitable expressions for the transformed splines $\mathcal{T} E$ and $\mathcal{S} E$ in 3.8 and 3.9. Such expressions may be found in terms of the integrals

$$
\begin{aligned}
& I_{\beta}\left(\omega ; \tau_{m}, \tau_{n}\right)=\int_{\tau_{m}}^{\tau_{n}}\left(\frac{\tau}{\tau_{m}}\right)^{\beta} \frac{\omega^{2} \tau^{2}}{1+\omega^{2} \tau^{2}} \frac{d \tau}{\tau}, \\
& J_{\beta}\left(\omega ; \tau_{m}, \tau_{n}\right)=\int_{\tau_{m}}^{\tau_{n}}\left(\frac{\tau}{\tau_{m}}\right)^{\beta} \frac{\omega \tau}{1+\omega^{2} \tau^{2}} \frac{d \tau}{\tau} .
\end{aligned}
$$

Specifically:

$$
\begin{aligned}
{\left[\mathcal{T} E\left(\tau \dot{\gamma}^{\alpha} ; \xi_{1}, \xi_{2}, \xi_{3}\right)\right](\omega) } & =-\frac{1}{2} \beta c_{1} \xi_{1}^{-1}\left[I_{\beta}\left(\omega ; \tau_{1}, \tau_{3}\right)-I_{-\beta}\left(\omega ; \tau_{1}, \tau_{3}\right)\right] \\
& -\frac{1}{2} \beta c_{2} \xi_{2}^{-1}\left[I_{\beta}\left(\omega ; \tau_{2}, \tau_{3}\right)-I_{-\beta}\left(\omega ; \tau_{2}, \tau_{3}\right)\right]
\end{aligned}
$$

and

$$
\begin{aligned}
{\left[\mathcal{S} E\left(\tau \dot{\gamma}^{\alpha} ; \xi_{1}, \xi_{2}, \xi_{3}\right)\right](\omega) } & =-\frac{1}{2} \beta c_{1} \xi_{1}^{-1}\left[J_{\beta}\left(\omega ; \tau_{1}, \tau_{3}\right)-J_{-\beta}\left(\omega ; \tau_{1}, \tau_{3}\right)\right] \\
& -\frac{1}{2} \beta c_{2} \xi_{2}^{-1}\left[J_{\beta}\left(\omega ; \tau_{2}, \tau_{3}\right)-J_{-\beta}\left(\omega ; \tau_{2}, \tau_{3}\right)\right]
\end{aligned}
$$

with $\tau_{m}=\xi_{m} \dot{\gamma}^{-\alpha}, \quad m=1,2,3$.

It must be noted that the above conversion also works in the reverse direction, from $G_{\perp}^{*}(\omega)$ to $G_{\|}^{*}(\omega)$. In this case the constants $\left\{c_{k m}, \xi_{k m}\right\}$ are determined by fitting the models 5.14 and 5.15 to the available OSR experimental data. It should also be emphasized that each set of parameters obtained by model-fitting is local to the range of shear-rates $\Gamma_{a b}$. Any flow curve can be separated into a set of local ranges $\Gamma_{a b}$ in which the power index, $-\alpha$, is effectively a constant. Within such a range, a PSR (or OSR) experiment need be conducted at only one representative shear-rate. Finally, it should be noted that if $\beta$ is an integer multiple of $\frac{1}{2}$ or $\frac{1}{3}$, the integrals 5.16 and 5.17 may be expressed in closed form in terms of elementary functions. Otherwise, they are readily computed by numerical quadratures. 


\subsection{A model example.}

We illustrate the theory presented above by considering a model example. The parallel response spectrum consists of a single discrete mode for the linear spectrum, plus a single Dirac triplet for the rate-dependent part. The rate-dependent parts of the parallel and orthogonal response spectra are scaled versions of the spectra shown in Figure 1. Viewed as a function of $\ln (\tau)$, the spectra shift to the left as shear-rate increases. In Figure 2 are shown log-log plots of $\left|G_{\|}^{\prime}(\omega)\right|$ obtained from 5.12 for three different shear-rates. As the shear-rate increases, the profiles shift to the right, and negative parts of $G_{\|}^{\prime}(\omega)$ become apparent. In Figure 3 are shown the corresponding log-log plots of $G_{\|}^{\prime \prime}(\omega)$ obtained from 5.13 for the same three different shear-rates. $G_{\|}^{\prime \prime}(\omega)$ has no negative values for this model example, even when extrapolated beyond the frequency range shown.

The converted $G_{\perp}^{\prime}$ and $G_{\perp}^{\prime \prime}$, obtained from equations 5.14 and 5.15 are shown in Figures 4 and 5, respectively. The frequency range in Figure 4 has been extended to demonstrate that the orthogonal and parallel moduli converge to the same plateau modulus at high frequency. Model 5.12 predicts the same plateau modulus for all shear rates in the local range $\Gamma_{a b}$.

\section{Limitations}

There is a wide range of constitutive models available which describes nonlinear response in superposition rheometry. It is important to note that the theory and results derived in this paper hold for the Lodge-type model (3.1). Whereas it may be anticipated that similar results hold for other models, rigorous conclusions pertaining to other constitutive models must await separate investigation. In particular, the theory and results are presented in the context of weak nonlinear response, and may well require modification in the case of strong nonlinear response. For example, Metri and Briels [17] indicate, on the basis of Brownian dynamics simulation, that the Kramers-Krönig relations for OSR can break down under conditions of strong nonlinear response.

The solution class studied in section 4.1 is for finite ranges of shear-rates strictly greater than zero. Different solution classes are available in the limit $\dot{\gamma} \rightarrow 0$, and asymptotic matching principles may be required to present a complete picture. Furthermore, numerical techniques may be required if the discrete and semi-discrete spectral representations in section 5.2 are replaced by fully continuous representations.

Finally, it should be clear that this is essentially a theoretical paper which begins to explore the nature of parallel response spectra and techniques of interconversion. We propose to follow this initial exploration with future investigations into other constitutive models, and compare their use in interconverting between experimental data available in the literature. 


\section{Conclusions}

In parallel superposition rheometry, the problem of how to interpret and utilize dynamic moduli derived from Lodge-type models has been unresolved since the seminal paper of Yamamoto in 1971 on rate-dependent relaxation spectra. It has been assumed many times in the rheological literature that the parallel dynamic moduli derived by Yamaoto do not satisfy the classical Kramers-Krönig relations. In this paper we have shown that these assumptions are false, i.e. that these parallel dynamic moduli do satisfy the classical Kramers-Krönig relations. We have also shown how to interpret parallel response spectra in Lodge-type models by relating them to their orthogonal counterparts. Special bases have been introduced which enable the interconversion of parallel and orthogonal response spectra, and of parallel and orthogonal dynamic moduli, in both directions.

\section{Acknowledgements}

We are grateful for the comments of three anonymous referees which have helped us improve the original manuscript. DJC acknowledges the support of EPSRC through the Centre for Innovative Manufacturing in Large Area Electronics (CIMLAE - EP/K03099X/1) and the EPSRC platform grant in Engineering Blood Diagnostics (EP/N013506/1). ARD acknowledges the support of EPSRC through the Inverse Problems Net work grant EP/P005985/1.

\section{References}

1. H. Booij, Influence of superimposed steady shear flow on the dynamic properties on non-Newtonian fluids i: Measurements on non-newtonian solutions, Rheologica Acta 5 (1966) 215-221.

2. I. Macdonald, R. Bird, Complex modulus of concentrated polymer solutions in steady shear, Journal of Physical Chemistry 70 (1966) 2068-2069.

3. J. Dealy, Official nomenclature for material functions describing the response of a viscoelastic fluid to various shearing and extensional deformations, Journal of Rheology 39 (1995) 253-265.

4. D. Curtis, N. Badiei, A. Hoder, J. Claypole, D. Deganello, M.R. Brown, M.J. Lawrence, P.A. Evans, P.R. Williams, K. Hawkins, Assessment of the stress relaxation characteristics of critical gels formed under unidirectional shear flow by controlled stress parallel superposition rheometry, Journal of Non- Newtonian Fluid Mechanics 222 (2015) 227-233.

5. N. Badiei, A.M, Sowedan, D.J. Curtis, M.R. Brown, M.J.Lawrence, A.I. Campbell, A. Sabra, P.A. Evans, J.W. Wiesel, I.N. Chernysh, C. Nagaswami, P.R. Williams,K. Hawkins, Effects of unidirectional on shear stresses on the formation, fractal microstructure and rigidity of incipient whole blood clots and fibrin gels., Clinical Haemorheology and Microcirculation 60 (2015) 451-464. 
6. A. Holder, J. Claypole, T. Claypole, P. Cooper, P.R. Williams, D.J. Curtis, Fourier transform controlled stress parallel superposition (ft-csps): Validation and application in processing printable functional materials, Physics of Fluids 30 (2018) 077105.

7. V. Anderson, J. Pearson, J. Sherwood, Oscillation superimposed on steady shearing: Measurements and predictions for wormlike micellar solutions, Journal of Rheology 50 (2006) 771-796.

8. E. Somma, Parallel superposition in entangled polydisperse polymer melts: Experiments and theory, Journal of Rheology 51 (2007) 987-1005.

9. P. Boukany, W.-Q.Wang, Nature of steady flow in entangled fluids revealed by superimposed small amplitude oscillatory shear, Journal of Rheology 53 (2009) 1425-1435.

10. J. Vermant, L.Walker, P. Moldenaers, J. Mewis, Orthogonal versus parallel superposition measurements, Journal of Non-Newtonian Fluid Mechanics 79 (1998) 173-189.

11. G. Colombo, K. Sunhyung, T. Schweizer, B. Schroyen, C. Clasen, J. Mewis, J. Vermant, Superposition rheology and anisotropy in rheological properties of sheared colloidal gels, Journal of Rheology 61 (2017) 1035-1048.

12. J. Vermant, P. Moldenaers, J. Mewis, M. Ellis, R. Garritano, Orthogonal superposition measurements using a rheometer equipped with a force rebalanced transducer, Rev. Sci. Instruments 68 (1997) 4090.

13. J. Mewis, N. Wagner, Colloidal Suspension Rheology, 1st Edition, Cambridge University Press, 2013.

14. M. Yamamoto, Rate-dependent relaxation spectra and their determination, Trans. Soc. Rheol. 15 (1971) 331-344.

15. J.C.Saut, D.D. Joseph, Fading memory, Archive for Rational Mechanics and Analysis, 81 (1993) 53-95.

16. S.N. Bernstein, Sur les fonctions absolument monotones, Acta Mathematica, 52 (1928) 1-66.

17. V. Metri, W.J. Briels, Brownian dynamics investigation of the Boltzmann superposition principle for orthogonal superposition rheology, J. Chem. Phys. 150, 014903 (2019); doi: 10.1063/1.5080333.

18. SH. Kim, J.Mewis, C. Clasen, J. Vermant, Superposition rheometry of a wormlike micellar fluid, Rheol. Acta 52 (2013) 727-740.

19. N.W. Tschoegl, (1989), The Phenomenological Theory of Linear Viscoelastic Behaviour (Berlin Heidelberg: Springer-Verlag). 


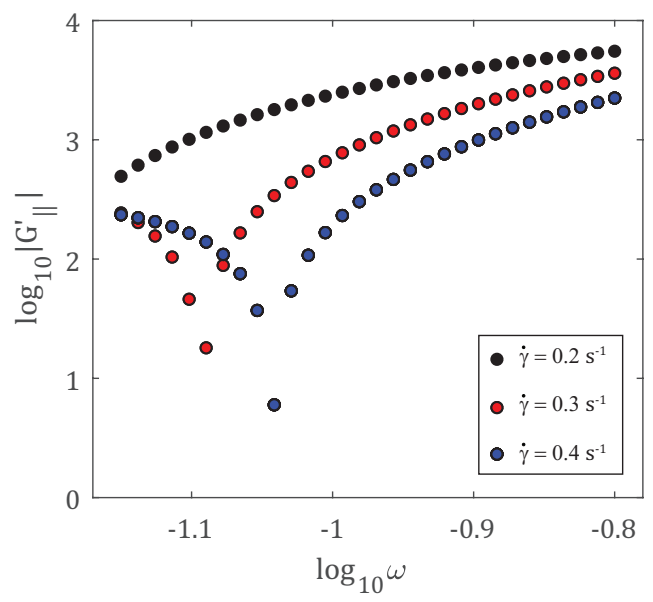

Figure 2: Model data for $\left|G_{\|}^{\prime}(\omega)\right|$ based on equation 5.12 for three shear-rates $\left(\dot{\gamma}_{1}, \dot{\gamma}_{2}, \dot{\gamma}_{3}\right)=$ $(0.2,0.3,0.4)$. Single linear mode $\lambda c_{0}=2.5 \times 10^{4}, \tau_{0}=4$. Single Dirac triplet with parameters $\beta=1,\left(c_{1}, c_{2}, c_{3}\right)=(-1,6.4,-5.4) \times 10^{4},\left(\xi_{1}, \xi_{2}, \xi_{3}\right)=(1,2,3)$.

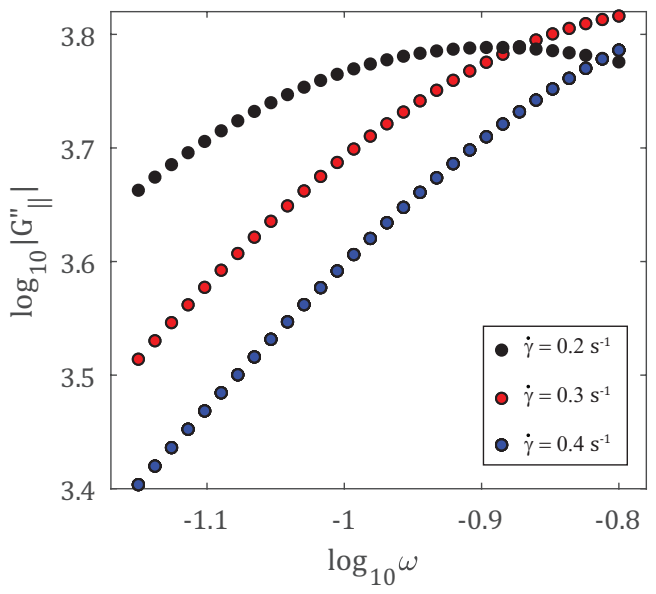

Figure 3: Model data for $G_{\|}^{\prime \prime}(\omega)$ based on equation 5.13 for three shear-rates $\left(\dot{\gamma}_{1}, \dot{\gamma}_{2}, \dot{\gamma}_{3}\right)=$ $(0.2,0.3,0.4)$. Single linear mode $\lambda c_{0}=2.5 \times 10^{4}, \tau_{0}=4$. Single Dirac triplet with parameters $\beta=1,\left(c_{1}, c_{2}, c_{3}\right)=(-1,6.4,-5.4) \times 10^{4},\left(\xi_{1}, \xi_{2}, \xi_{3}\right)=(1,2,3)$. 


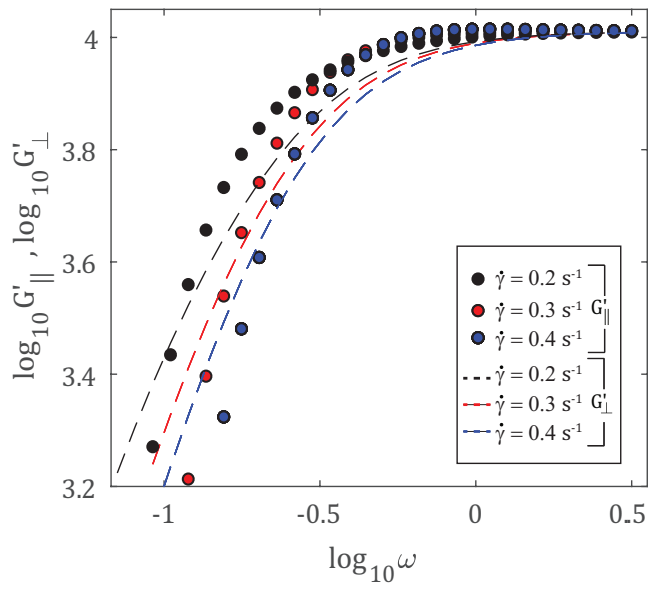

Figure 4: Model data. Comparison of $G_{\|}^{\prime}(\omega)$ in Figure 2 with corresponding $G_{\perp}^{\prime}(\omega)$ at same shear-rates.

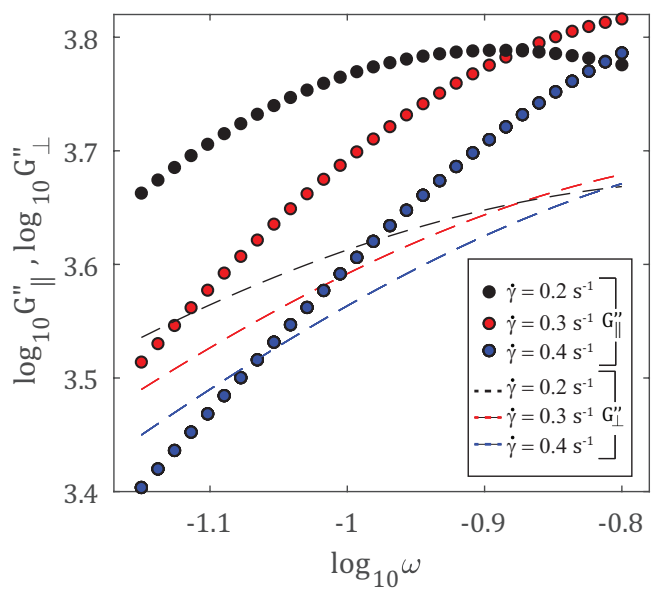

Figure 5: Model data. Comparison of $G_{\|}^{\prime \prime}(\omega)$ in Figure 3 with corresponding $G_{\perp}^{\prime \prime}(\omega)$ at same shear-rates. 